\title{
Síndrome De Down E Cardiopatia Congênita: Estado Nutricional Em Diferentes Faixas Etárias
}

\author{
Dornelles, I.K.; Foscarini, A.C.; Sica, C.A.; Pellanda, L.C.;
}

Apresentador: Ismael Kirst Dornelles

\section{Resumo}

Introdução: O sobrepeso e a obesidade estão, frequentemente, presentes em portadores de síndrome de Down (SD). Contudo, sabe-se que tais indivíduos possuem velocidade de ganho de peso, desde a infância até a adolescência, semelhante a dos não portadores de $\mathrm{SD}$, não ocorrendo o mesmo em relação à velocidade de ganho estatural - a qual é diminuída entre esses indivíduos. Com isso, objetivamos avaliar condição antropométrica, estado nutricional, aspectos dietéticos, frequência de atividade física e de fatores de risco para cardiopatia isquêmica em portadores de SD com cardiopatia congênita (CC). Métodos: Estudo transversal, envolvendo crianças e adolescentes de 2 a 18 anos de idade, portadores de SD e CC, atendidos no Ambulatório de Cardiologia Pediátrica do Instituto de Cardiologia do Rio Grande do Sul. Foram estudados: valor energético total (VET), circunferências (cintura e quadril), pressão arterial, perfil lipídico, atividade física diária e frequência de fatores de risco para cardiopatia isquêmica. As prevalências foram descritas sob a forma de proporções com respectivos intervalos de confiança de 95\%. Para análises estatísticas, foi utilizado o programa SPSS versão 19.0. Resultados: Foram avaliadas 68 crianças e adolescentes portadores de SD com CC. No grupo I (entre 2 e 10 anos) encontrou-se uma prevalência de 5,4\% com baixa estatura para idade, $68,4 \%$ eutróficos e 13,2\% com sobrepeso. No grupo II (idade igual ou maior a 10 até 18 anos) encontrou-se uma prevalência de 23,3\% com baixa estatura para idade e 76,6\% eutróficos. A prevalência dos fatores de risco foi maior para hipertensão arterial, doença arterial coronariana e dislipidemia. Em relação aos lipídios, houve um equilíbrio entre os participantes que consomem quantidade abaixo da recomendação e a recomendada. A maioria dos participantes foi classificada como ativos ou muito ativos quanto à prática de atividade física. Conclusão: As crianças e adolescentes com SD e CC encontram-se, em sua maioria, com peso adequado para idade, assim como estatura para idade, segundo as curvas propostas por Cronk et al. (1988), em desacordo com o paradigma de que estes indivíduos, geralmente, teriam um estado nutricional desfavorável com sobrepeso e baixa estatura para idade. Podemos associar as mudanças no estado nutricional destes indivíduos devido ao fortalecimento no núcleo familiar - são atendidos por profissionais das diversas áreas da saúde, onde possuem um acompanhamento adequado para suas características.

\section{Referência:}

Dornelles, I.K.; Foscarini, A.C.; Sica, C.A.; Pellanda, L.C.;. Síndrome De Down E Cardiopatia Congênita: Estado Nutricional Em Diferentes Faixas Etárias. In: II Congresso Brasileiro de Medicina Hospitalar - II CBMH [= Blucher Medical Proceedings, vol.1, num.5] São Paulo: Editora Blucher, 2014. p.37

DOI 10.5151/medpro-II-cbmh-030 